DOI: $10.5965 / 223811711612017070$

\title{
Vacas F1 Holandês x Zebu no terço final de gestação apresentam adaptação fisiológica quando criadas no ambiente semiárido
}

F1 Holstein $x$ Zebu Cows in late pregnancy show physiological adaptation when raised in semiarid environment

Thamara Amaral Diniz, Cinara da Cunha Siqueira Carvalho*, Heberth Christian Ferreira, Anna Luísa de Oliveira Castro, Kátia Cristiane Borges Pereira, Maria Cecília Magalhães Gonçalves, Thaís Emanuele Soares e Jordana Carvalho Menezes

Recebido em 16/07/2016 / Aceito em 11/10/2016

\section{RESUMO}

O trabalho teve como objetivo verificar a adaptabilidade de vacas F1 Holandês x Zebu no terço final de gestação, criadas no semiárido mineiro, por meio das respostas fisiológicas. Foram utilizadas 10 vacas, todas de quarta gestação. Para caracterizar o ambiente climático foram realizadas coletas diárias de temperatura do ar, umidade relativa do ar e velocidade do vento, para possibilitar o cálculo do Índice de Temperatura de Globo e Umidade (ITGU) e da Carga Térmica Radiante (CTR). Os parâmetros fisiológicos analisados foram: frequência respiratória, temperatura retal e temperatura da superfície corporal, às 10:00 e 15:00 horas. Os testes de adaptabilidade utilizados foram: Índice de tolerância ao calor (ITC) e coeficiente de tolerância ao calor (CTC). O ambiente climático esteve fora das condições de conforto térmico no período da tarde. Houve diferença significativa $(\mathrm{p}<0,05)$ para os valores de parâmetros fisiológicos, contudo, essas respostas estiveram dentro da faixa de conforto recomendados para animais mestiços. Os testes de adaptabilidade confirmaram que os animais apresentam adaptação ao ambiente do semiárido mineiro, apresentando diferenças $(\mathrm{p}<0,05)$, de CTC entre o horário das 10:00 h $(2,52)$ e 15:00 h $(2,64)$, e o ITC apresentou uma média de 9,64, valor aproximado do índice 10. Conclui-se que, as vacas F1 Holandês x Zebu no terço final de gestação são adaptadas ao clima semiárido mineiro, por não alterar o comportamento fisiológico diante das adversidades do ambiente climático.

PALAVRAS-CHAVE: agricultura familiar, ambiência, bovino leiteiro, heterose.

\section{ABSTRACT}

This study aimed to verify the adaptability of F1 Holstein $\mathrm{x}$ Zebu cows during the late pregnancy third, raised in the semiarid region of Minas Gerais, by means of physiological responses. A total of ten cows were used, all in fourth pregnancy. To characterize the climatic environment, daily collections of air temperature, air relative humidity and wind speed were carried out to enable the calculation of the Globe Temperature and Humidity Index (GTHI) and Radiant Thermal Load (RTL). The physiological parameters analyzed were: respiratory frequency, rectal temperature and surface body temperature, at $10 \mathrm{am}$ and $3 \mathrm{pm}$. The adaptability tests used were Heat Tolerance Index (HTI) and Heat Tolerance Coefficient (HTC). The climatic environment was out of thermal comfort in the afternoon. There were significant differences $(p<0.05)$ for the values of the physiological parameters. However, these responses were within the comfort range recommended for crossbred animals. The adaptability tests confirmed that the animals show adaptation to the semiarid environment of Minas Gerais, showing differences $(\mathrm{p}<0.05)$ on HTC between 10 am (2.52) and $3 \mathrm{pm}$ (2.64), and the HTI showed an average of 9.64, a value that is very close to the index 10. It is concluded that the F1 Holstein x Zebu cows in the late pregnancy third are adapted to the climate in the semiarid region of Minas Gerais, by not altering their physiological behavior in face of the adversities of the climatic environment.

KEYWORDS: family farming, ambience, dairy cattle, heterosis.

Universidade Estadual de Montes Claros, Montes Claros, MG, Brasil.

*Autor para correspondência <cinarasiqueira@yahoo.com.br> 


\section{INTRODUÇÃO}

O Estado de Minas Gerais é considerado o principal produtor de leite no Brasil com 9,37 bilhões de litros, o que corresponde a $77,0 \%$ de toda a produção da Região Sudeste e a $26,6 \%$ do total da produção nacional (IBGE 2015). No contexto do cenário mineiro, a região Norte de Minas, apresenta uma produtividade limitada, principalmente em função da seca, porém é ainda responsável pela produção de 412 mil litros de leite, fundamentada em grande parte, pela agricultura familiar (IBGE 2012). A resposta produtiva dos animais está condicionada a fatores como a nutrição, sanidade e o ambiente onde estão inseridos. Assim, o clima destaca-se por influenciar na ocorrência do cio, manutenção da gestação e produção satisfatória de leite (PIRES et al. 2010, TAO et al. 2012).

Contudo, considerando as fases específicas da criação de bovinos leiteiros, sabe-se que durante o terço final da gestação, ocorre um aumento da temperatura corporal interna devido ao crescimento fetal, elevando a pressão interna nos órgãos digestivos e diminuição do espaço ocupado pelos alimentos. Essa condição leva à redução do consumo de matéria seca, predispondo o animal a um balanço energético negativo, alteração nos parâmetros fisiológicos, comportamentais, além de ocorrer a redução no peso do feto, funções imunológicas e produção de leite (AMARAL et al. 2010, TAO et al. 2012).

Neste sentido, o uso de animais mestiços em regiões de clima semiárido pode ser uma alternativa em função da união da rusticidade do animal zebu com a produtividade do holandês (PIRES et al. 2010, RUAS et al. 2010). Dessa forma, objetivouse verificar a adaptabilidade, bem como as respostas fisiológicas de diferentes cruzamentos de vacas F1 Holandês x Zebu, no terço final da gestação, criadas no semiárido mineiro.

\section{MATERIAL E MÉTODOS}

O experimento foi conduzido na Fazenda Experimental da Universidade Estadual de Montes Claros - UNIMONTES, no município de Janaúba, MG, no período de novembro a dezembro de 2015. A área experimental está localizada nas coordenadas geográficas $43^{\circ} 16^{\prime} 8,2^{\prime \prime} \mathrm{W}$ e $15^{\circ} 49^{\prime} 51,5^{\prime \prime} \mathrm{S}$, com altitude média de aproximadamente $540 \mathrm{~m}$. A pluviosidade média da região é de $870 \mathrm{~mm}$, a temperatura média anual de 24 a $28{ }^{\circ} \mathrm{C}$, a umidade relativa média de $65 \%$ e o tipo de clima Aw caracterizando-se tropical chuvoso e de savana com inverno seco, segundo a classificação de Köppen.

Foram utilizadas 10 vacas F1, com peso médio de $500 \mathrm{Kg}$, todas na quarta gestação e compreendendo o terço final da gestação. As 10 vacas F1 são oriundas do cruzamento proveniente de touro holandês com vacas zebuínas, constituindo animais de grupos genéticos com $100 \%$ de heterose, sendo eles: 3 animais F1: Holandês (50\%) x Gir (50\%); 4 animais F1: Holandês (50\%) x Nelore (25\%) x Gir $(25 \%)$ e 3 animais F1: Holandês (50\%) x Nelore (50\%).

O período experimental teve duração de 13 dias, e durante a semana os animais eram mantidos no curral recebendo cana-de-açúcar moída misturado com concentrado e água à vontade, e nos finais de semana, estas ficavam no pasto, que possuía como fonte de alimento Brachiaria Brizantha cv. Marandu ou Tifton 85 com livre acesso ao curral para consumo de água.

Para caracterizar o ambiente climático foram realizadas coletas diárias das variáveis climáticas: temperatura do ar, umidade relativa do ar e velocidade do vento. Para a obtenção das variáveis climáticas utilizou-se 2 dataloggers de leitura contínua da marca Extech, modelo RHT10, programados para coletar os dados a cada 10 minutos. Um datalogger foi programado para coletar os dados da temperatura e umidade relativa do ar e o outro, colocado no interior do globo, para determinar a temperatura de globo negro. Os equipamentos foram instalados a uma altura de 1,60 metros.

De posse dessas variáveis climáticas coletadas calculou-se o Índice de Temperatura e Umidade (ITGU) proposto por BUFFINGTON et al. (1981) obtido com a seguinte expressão.

$$
\mathrm{ITGU}=\mathrm{Tgn}+0,36 \mathrm{Tpo}+41,5 \quad \text { eq. } 1
$$

Onde:

Tpo $=$ Temperatura do ponto de orvalho $\left({ }^{\circ} \mathrm{C}\right)$;

Tgn $=$ Temperatura do globo negro $\left({ }^{\circ} \mathrm{C}\right)$.

A velocidade do vento foi obtida por meio do uso do anemômetro digital portátil, modelo AD 250, marca Instrutherm. Além do ITGU, foi calculado também a carga térmica radiante (CTR) que incide sobre os animais. 


$$
\mathrm{CTR}=\mathrm{S}(\mathrm{TRM})^{4} \quad \text { eq. } 2 \text { Em que: }
$$

$\mathrm{CA}=$ coeficiente de adaptabilidade;

Em que:

$\mathrm{CTR}=$ carga térmica de radiação, em $\mathrm{W} \cdot \mathrm{m}^{-2}$; $\mathrm{S}=$ constante Stefan - Boltzmann $\left(5,67 \times 10^{-8} \mathrm{~W} \cdot \mathrm{m}^{-}\right.$ $\left.{ }^{2} \mathrm{~K}^{-4}\right)$.

A Temperatura Radiante Média (TRM) foi obtida segundo a equação:

$$
\mathrm{TRM}=100 \sqrt[4]{2,51 \sqrt{V(\operatorname{Tgn}-T b s)+\left(\frac{\operatorname{Tgn}}{100}\right)^{4}}} \text { eq. } 3
$$

Em que:

$\mathrm{TRM}=$ temperatura radiante média, em $\mathrm{k}$;

$\mathrm{V}=$ velocidade do vento, em $\mathrm{m} / \mathrm{s}$;

Tgn $=$ temperatura do globo negro $\left({ }^{\circ} \mathrm{C}\right)$;

Tbs = temperatura de bulbo seco (do ar), em $\mathrm{k}$.

Os parâmetros analisados foram: frequência respiratória (FR), temperatura retal (TR) e temperatura da superfície corporal (TSC). Para obter esses valores, os animais foram manejados até o brete nos horários de 10:00 e 15:00 horas.

A frequência respiratória foi medida por meio da visualização dos movimentos do flanco do animal, durante 15 segundos, o valor obtido foi multiplicado por quatro para obter o número de movimentos por minuto. A temperatura retal foi mensurada com auxílio de um termômetro clínico digital, inserido diretamente no reto, permanecendo até o instante em que o sensor emitia um sinal sonoro. A temperatura da superfície corporal foi medida no dorso do animal, utilizando um termômetro infravermelho.

Os resultados obtidos para TR e FR foram utilizados para cálculo do Índice de Tolerância ao Calor e Coeficiente de Tolerância ao Calor (CTC), por meio das seguintes fórmulas descritas a seguir:

$$
\mathrm{ITC}=10-(\mathrm{TR} 1-\mathrm{TR} 2) \quad \text { eq. } 4
$$

Em que:

ITC = índice de tolerância ao calor;

TR 1 = temperatura retal medida às 10 horas;

$\mathrm{TR} 2=$ temperatura retal medida às 15 horas.

Coeficiente de Tolerância ao Calor - Fórmula do teste de Benezra:

$$
\mathrm{CA}=\frac{\mathrm{TR}}{38,3}+\frac{\mathrm{FR}}{23}
$$

eq. 5
$\mathrm{TR}=$ temperatura retal, $\mathrm{em}{ }^{\circ} \mathrm{C}$;

$\mathrm{FR}=$ frequência respiratória, em movimentos respiratórios/minuto.

O experimento foi conduzido utilizando-se o delineamento inteiramente casualizado (DIC) com esquema fatorial $3 \times 2$, sendo três grupos genéticos (Holandês x Gir, Holandês x Nelore x Gir , Holandês x Nelore) e dois horários (10 h e $15 \mathrm{~h}$ ), com 10 repetições. Os três grupos genéticos estudados se mostraram semelhantes, portanto, foram unificados e as variáveis apresentadas e discutidas em função dos 2 horários sobre o comportamento fisiológico dos animais. As variáveis foram submetidas à análise de variância utilizando o programa estatístico SISVAR ${ }^{\circledR}$ (FERREIRA 2008), e quando o teste $\mathrm{F}$ foi significativo, as médias dos tratamentos foram comparadas pelo teste de Tukey $(\mathrm{p}<0,05)$.

\section{RESULTADOS E DISCUSSÃO}

Houve diferença significativa $(\mathrm{p}<0,05)$ para os horários analisados, sendo que os menores valores de temperatura do ar foram verificados às 10:00 h, com exceção da umidade relativa do ar. De acordo com SILVA (2000), a faixa de termoneutralidade para animais mestiços está compreendida entre $7{ }^{\circ} \mathrm{C}$ e $35^{\circ} \mathrm{C}$. Durante o período da manhã, o valor verificado estava dentro da faixa recomendada, porém às 15:00h, a temperatura do ar estava elevada (Tabela 1).

Em ambos os horários avaliados, os valores de UR podem ser considerados ideais às $10 \mathrm{~h}(50,8 \%)$

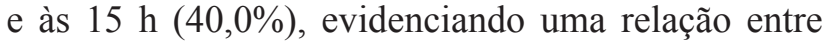
a maior temperatura do dia com a menor umidade relativa, contudo, sem afetar o conforto térmico ambiental. A junção da temperatura do ar com a umidade relativa informa a faixa de conforto térmico ambiental a qual os animais estão expostos. De acordo com BUFFINGTON et al. (1981), valores de ITGU de até 74 definem condição de conforto no ambiente, de 74 a 78 é sinal de alerta, de 79 a 84 é sinal de perigo e, acima de 84 é considerado sinal de emergência. Os valores médios de ITGU obtidos caracterizaram o ambiente climático em situação perigosa $(10 \mathrm{~h})$ e de emergência (15 h).

A carga térmica radiante, dada em forma de energia (W) incidente sobre a área corporal do animal $\left(\mathrm{m}^{2}\right)$ foi diferente $(\mathrm{p}<0,05)$ entre os horários 
avaliados, obtendo valores de 457,7 W.m ${ }^{-2}$ às $10 \mathrm{~h}$ e de 480,7 W.m ${ }^{-2}$ às 15 h. Animais criados a pasto estão mais expostos à radiação e a absorção de calor, assim, de acordo com SILVA (2000), quanto menor o valor de CTR, maior o bem estar para os animais. Portanto, em todos os horários, os valores médios da CTR se mantiveram abaixo do valor médio verificado por FAÇANHA et al. (2010), que encontraram valor máximo de CTR médio no verão de $768,7 \mathrm{~W} \cdot \mathrm{m}^{-2} \mathrm{em}$ vacas da raça Holandesa no semiárido cearense.

Os animais ruminantes criados sob condições térmicas desfavoráveis, tendem a alterar o incremento calórico da atividade voluntária da fermentação ruminal, reduzindo a ingestão e digestão do alimento, o metabolismo, a absorção de nutrientes, além de ajustarem as respostas fisiológicas, comportamentais e produtivas no intuito de se adequarem ao ambiente de criação (SANTOS et al. 2006, SOUZA et al. 2007, NÓBREGA et al. 2011).

Os valores médios dos parâmetros fisiológicos (TSC, FR e TR) foram semelhantes $(p>0,05)$ para os grupos genéticos, havendo diferença $(p<0,05)$ na avaliação dos horários, contudo, todos os valores estão dentro da faixa de normalidade para a espécie (Tabela 2).

FERREIRA et al. (2006) determinaram em vacas $\mathrm{F} 1$ durante o verão, $\mathrm{TSC}$ de $27,7^{\circ} \mathrm{C}$ no período da manhã, e TSC de $46,35{ }^{\circ} \mathrm{C}$ no período da tarde. No presente trabalho, a TSC avaliada em função dos grupos genéticos, apresentaram valores médios intermediários aos verificados por FERREIRA et al. (2006), inclusive no período da tarde $\left(37,7{ }^{\circ} \mathrm{C}\right)$ quando a TSC foi superior à manhã $\left(35^{\circ} \mathrm{C}\right)$. Segundo CUNNINGHAM (1999), a temperatura de superfície corporal sofre alterações em função das condições climáticas e fisiológicas (vascularização e evaporação pelo suor), contudo, as trocas de calor com o ambiente por meio da pele (radiação, condução e convecção) é uma das formas dos animais obterem a homeotermia (CUNNINGHAM 1999).

A frequência respiratória também é muito usada para avaliar o estresse calórico dos animais, em bovinos adultos pode variar entre 12 a 60 movimentos por minutos (GAUGHAN et al. 1999, FERREIRA et al. 2006). Neste trabalho, os valores verificados em função dos grupamentos genéticos e horários, estão

Tabela 1 - Valores médios de Temperatura do ar (Tar, ${ }^{\circ} \mathrm{C}$ ), Umidade Relativa do ar (UR, \%), Índice de temperatura do Globo e Umidade (ITGU) e Carga Térmica Radiante (CTR, W.m ${ }^{-2}$ ), referentes ao período de análise dos testes de adaptabilidade.

Table 1 - Average Air Temperature values (Tar, ${ }^{\circ} \mathrm{C}$ ) Relative Humidity (UR,\%), Globe and Humidity Temperature Index (ITGU) and Radiant Thermal Load (CTR, W.m- $\left.{ }^{2}\right)$ for the period of analysis of adaptability tests.

\begin{tabular}{lcccc}
\hline Horário & Tar $\left({ }^{\circ} \mathrm{C}\right)$ & UR $(\%)$ & ITGU & CTR $\left(\mathrm{W} \cdot \mathrm{m}^{-2}\right)$ \\
\hline $10: 00$ & $32,8 \mathrm{a}$ & $50,8 \mathrm{a}$ & $83,1 \mathrm{a}$ & $457,7 \mathrm{a}$ \\
$15: 00$ & $37,8 \mathrm{~b}$ & $40,0 \mathrm{~b}$ & $87,6 \mathrm{~b}$ & $480,7 \mathrm{~b}$ \\
\hline
\end{tabular}

Médias seguidas pelas mesmas letras nas colunas não diferem entre si pelo de teste de Tukey $(\mathrm{p}<0,05)$.

Tabela 2 - Valores médios de temperatura de superfície corporal (TSC), frequência respiratória (FR) e temperatura retal (TR) em função dos cruzamentos genéticos e horários avaliados.

Table 2 - Mean Body Surface Temperature values (TSC), Respiratory Rate (FR) and Rectal Temperature (TR) on the basis of genetic crosses and evaluated times.

\begin{tabular}{lccccc}
\hline \multirow{2}{*}{ Respostas fisiológicas } & \multicolumn{3}{c}{ Cruzamentos } & \multicolumn{2}{c}{ Horários } \\
\cline { 2 - 6 } & H x Gir & H x Nel & H x Nel x Gir & $10: 00$ & $15: 00$ \\
\hline TSC $\left({ }^{\circ} \mathrm{C}\right)$ & $36,2^{\text {ns }}$ & $36,5^{\text {ns }}$ & $36,5^{\text {ns }}$ & $35,0 \mathrm{a}$ & $37,7 \mathrm{~b}$ \\
FR $\left(\right.$ mov.min $\left.^{-1}\right)$ & $36,0^{\text {ns }}$ & $36,1^{\text {ns }}$ & $36,5^{\text {ns }}$ & $35,1 \mathrm{a}$ & $37,4 \mathrm{~b}$ \\
TR $\left({ }^{\circ} \mathrm{C}\right)$ & $38,5^{\text {ns }}$ & $38,5^{\text {ns }}$ & $38,6^{\text {ns }}$ & $38,2 \mathrm{a}$ & $38,8 \mathrm{~b}$
\end{tabular}

Médias seguidas pelas mesmas letras nas colunas não diferem entre si pelo de teste de Tukey $(p<0,05)$.

ns não significativo. 
de acordo com os valores da literatura.

SILVA et al. (2009) avaliando os efeitos da disponibilidade de sombra sobre os parâmetros fisiológicos de vacas da raça Pitangueiras no estado de Pernambuco, com condições de ITGU médio de 83 no período da manhã e de 85 à tarde, verificaram frequência respiratória de 46,65 e 51,85 mov. $\mathrm{min}^{-1}$, respectivamente. Contudo, no presente trabalho mesmo com os valores de ITGU e CTR superiores no período da tarde (Tabela 1), a frequência respiratória das vacas F1 foram menores do que os valores verificados por SILVA et al. (2009), indicando que o ambiente climático de criação não exigiu o acionamento do sistema de termorregulação dos animais.

A temperatura retal é o principal índice para avaliação da adaptação ao ambiente climático, pois quando a TR é elevada, entende-se que os mecanismos de liberação de calor foram insuficientes para manter a homeotermia. AMARAL et al. (2010) avaliando vacas no terço final da gestação, verificaram no período da manhã TR de $38,8{ }^{\circ} \mathrm{C}$ e a tarde, TR de $39,2{ }^{\circ} \mathrm{C}$. Essa condição foi verificada neste trabalho, tanto na avaliação dos cruzamentos genéticos, bem como, para os horários. PERISSINOTTO et al. (2009) relataram que quando a TR encontra-se na faixa de
38,7 a $39,2^{\circ} \mathrm{C}$ e a frequência respiratória é menor ou igual a 54 mov.min ${ }^{-1}$, há um indicativo de estado de conforto térmico dos animais. Sendo essa, a condição verificada neste trabalho.

Os resultados de CTC apresentaram diferenças $(\mathrm{p}<0,05)$, entre o horário das $10 \mathrm{~h}(2,52)$ e $15 \mathrm{~h}(2,64)$, evidenciando que as vacas estavam adaptadas ao clima do ambiente (Tabela 3), mesmo com os valores de ITGU fora da condição de conforto. SOUZA et al. (2010) avaliando a relação entre o ambiente físico e respostas fisiológicas de novilhas de raça leiteira obteve os valores de CTC de 5,13, logo após a exposição à radiação solar direta, o que pode ser considerado um estresse severo, uma vez que o ideal é igual a 2.

O ITC varia de 0 a 10 , o que representa a capacidade dos animais de dissiparem o calor absorvido durante a exposição ao sol (BACCARI JÚNIOR 1990). Na Tabela 4, verifica-se que o ITC apresentou uma média de 9,64, valor aproximado do índice 10, indicando que os animais apresentaram uma boa capacidade de dissiparem o calor absorvido durante a exposição ao sol, ou seja, mostram a tolerância do animal frente às adversidades do meio.

SOUZA et al. (2010) observaram em novilhas leiteiras mestiças Holandês x Jersey, em Piracicaba,

Tabela 3 - Resultados do coeficiente de tolerância ao calor (CTC) de vacas F1 Holandês x Zebu no terço final de gestação.

Table 3 - Results of Heat Tolerance Coefficient (CTC) F1 HxZ cows in late pregnancy third.

\begin{tabular}{cc}
\hline Horário & CTC - Benezra \\
\hline $10: 00$ & $2,52 \mathrm{a}$ \\
$15: 00$ & $2,64 \mathrm{~b}$ \\
\hline
\end{tabular}

Médias seguidas por diferentes nas colunas diferem entre si pelo de teste de Tukey $(\mathrm{p}<0,05)$.

Tabela 4 - Resultados do Índice de Tolerância ao Calor (ITC) de vacas F1 Holandês x Zebu. Table 4 - Index Tolerance results when heat (IT) F1 HxZ cows.

\begin{tabular}{cccc}
\hline Animal & TRtarde $\left({ }^{\circ} \mathrm{C}\right)$ & TRmanhã $\left({ }^{\circ} \mathrm{C}\right)$ & ITC \\
\hline 1 & 38,9 & 38,2 & 9,7 \\
2 & 38,7 & 37,8 & 9,7 \\
3 & 38,7 & 37,9 & 9,8 \\
4 & 39 & 38,3 & 9,6 \\
5 & 39 & 38.2 & 9,4 \\
6 & 39,2 & 38,3 & 9,5 \\
7 & 38,8 & 38,3 & 9,7 \\
8 & 38,9 & 38,5 & 9,6 \\
9 & 38,7 & 38,3 & 9,7 \\
10 & 38,8 & 38,4 & 9,7 \\
\hline Média & 38,9 & 38,2 & 9,64 \\
\hline
\end{tabular}

74 Revista de Ciências Agroveterinárias, Lages, v.16, n.1, 2017 
SP, uma média para o ITC de 9,85. Bem como, SOUZA et al. (2007) que avaliaram a rusticidade da raça Sindi no semiárido paraibano, e verificaram índice de tolerância ao calor de 9,83 , atingindo quase a nota máxima preconizada pelo teste aplicado. De acordo com o último autor, o ITC próximo a 10 , pode ser entendido como uma forma matemática de expressar a resistência dos animais a uma determinada condição climática, garantindo sua exploração na região com maiores possibilidades de lucros.

\section{CONCLUSÃO}

Vacas F1 Holandês x Zebu durante o terço final de gestação apresentaram-se fisiologicamente adaptadas ao clima do semiárido mineiro, mesmo quando os índices climáticos estão acima da condição de conforto térmico.

\section{AGRADECIMENTOS}

\section{À FAPEMIG, CAPES, CNPq e UNIMONTES.}

\section{REFERÊNCIAS}

AMARAL BC et al. 2010. Heat stress abatement during the dry period influences prolactin signaling in lymphocytes. Domestic Animal Endocrinology 38: 38-45.

BACCARI JÚNIOR F. 1990. Métodos e técnicas de avaliação da adaptabilidade dos animais às condições tropicais. In: Simpósio internacional de bioclimatologia animal nos trópicos: pequenos e grandes ruminantes, Anais... Sobral: EMBRAPA-CNPC. p.9-17.

BUFFINGTON DE et al. 1981. Black globe humidity index (BGHI) as comfort equation for dairy cows. Transacofthe ASAE 24: 711-714.

CUNNINGHAM JG. 1999. Tratado de fisiologia veterinária. 2.ed. Rio de Janeiro: Guanabara Koogan. 454p. FAÇANHA DAE et al. 2010. Variação anual de características morfológicas e da temperatura de superfície do pelame de vacas da raça Holandesa em ambiente semiárido. Revista Brasileira de Zootecnia 39: 837-844.

FERREIRA $\mathrm{F}$ et al. 2006. Parâmetros fisiológicos de bovinos cruzados submetidos ao estresse calórico. Arquivo Brasileiro de Medicina Veterinária e Zootecnia 58: 732738.

FERREIRA DF. 2008. Programa Sisvar versão 5.1. Programa de análises estatísticas. Lavras: DEX/UFLA.

GAUGHAN JB et al. 1999. Heat tolerance of Boran and Tuli crossbred steers. Journal of Animal Science 77: 23982405.

IBGE - Instituto Brasileiro de Geografia e Estatística. 2012. Pesquisa Pecuária Municipal. Disponível em: http://www. milkpoint.com.br/seu-espaco/espaco-aberto/brasil-leiteirode-sul-a-norte-minas-gerais-90135n.asp. Acesso em: 06 abr. 2016.

IBGE - Instituto Brasileiro de Geografia e Estatística. 2015. Sidra: sistema IBGE de recuperação automática. Tab. 1086. Disponível em: <http://www.sidra.ibge.gov.br>. Acesso em: 11 abr. 2016.

NÓBREGA GH et al. 2011. A produção animal sob a influência do ambiente nas condições do semiárido nordestino. Revista Verde 6: 67-73.

PIRES MFA et al. 2010. Adaptação de animais mestiços em ambiente tropical. Informe Agropecuário 31: 30-38.

PERISSINOTTO M et al. 2009. Conforto térmico de bovinos leiteiros confinados em clima subtropical e mediterrâneo pela análise de parâmetros fisiológicos utilizando a teoria dos conjuntos fuzzy. Ciência Rural 39: 1492-1498.

RUAS JRM et al. 2010. Sistema de Produção de Leite com vacas F1 Holandês x Zebu. Informe Agropecuário 31: 6371.

SANTOS JRS et al. 2006. Respostas fisiológicas e gradientes térmicos de ovinos das raças Santa Inês, Morada Nova e de seus cruzamentos com a raça Dorper às condições do semiárido nordestino. Ciência e Agrotecnologia 30: 995-1001.

SILVA ECL et al. 2009. Efeitos da disponibilidade de sombra sobre o desempenho, atividades comportamentais e parâmetros fisiológicos de vacas da raça Pitangueiras. Acta Scientiarum Animal Science 31: 295-302.

SILVA RG 2000. Introdução à bioclimatologia animal. São Paulo: Nobel. 286p.

SOUZA BB et al. 2007. Parâmetros fisiológicos e índice de tolerância ao calor de bovinos da raça Sindi no semi-árido paraibano. Ciência e Agrotecnologia 31: 883-888.

SOUZA BB et al. 2010. Estudo do ambiente físico sobre as respostas fisiológicas de novilhas de raça leiteira. Agropecuária Científica do Semiárido 6: 59-65.

TAO S et al. 2012. Effect of late-gestation maternal heat stress on growth and immune function of dairy calves. Journal of Dairy Science 95: 7128-7136. 